\title{
Dynamic Routing for Emergency Vehicle by Collecting Real-Time Road Conditions
}

\author{
Ryo Katsuma, Shin Yoshida \\ Osaka Prefecture University, Osaka, Japan \\ Email: ryo-k@mi.s.osakafu-u.ac.jp
}

How to cite this paper: Katsuma, R. and Yoshida, S. (2018) Dynamic Routing for Emergency Vehicle by Collecting Real-Time Road Conditions. Int. J. Communications, Network and System Sciences, 11, 27-44. https://doi.org/10.4236/ijcns.2018.112003

Received: October 25, 2017

Accepted: February 25, 2018

Published: February 28, 2018

Copyright $\odot 2018$ by authors and Scientific Research Publishing Inc. This work is licensed under the Creative Commons Attribution International License (CC BY 4.0).

http://creativecommons.org/licenses/by/4.0/

\section{(c) (i) Open Access}

\begin{abstract}
When a natural disaster such as earthquake strikes, people go away to safety areas by cars. Some roads are broken and impenetrable by buildings falling down. Furthermore, some fixed infrastructures for radio communication may be broken. In order to quickly bring ambulances and fire trucks to their destinations, it is necessary to provide real-time road conditions to these emergency vehicles via ad-hoc networks. In this paper, we propose a method to periodically reconstruct a route for an emergency vehicle by collecting real-time road conditions via vehicle ad-hoc networks. As simulation results, we confirmed that the proposed method collects $12 \%$ more road conditions than existing method. We also confirmed that the proposed method achieves $10 \%$ faster arrival than the existing method.
\end{abstract}

\section{Keywords}

Emergency Vehicle, Routing, Disaster, Vehicle ad-Hoc Networks

\section{Introduction}

Recently, many efforts have been devoted for traffic systems with radio communication such as VICS (Vehicle Information and Communication Systems) and ETC (Electronic Toll Collection systems). Especially, significant attention has been paid to two network architectures: V2I (Vehicle to Infrastructure) and V2V (Vehicle to Vehicle). A V2I architecture enables a vehicle and a roadside device to communicate with each other. However, the network may be cut due to failure of roadside devices in a disaster. On the other hand, a V2V architecture makes inter-vehicle connection without infrastructures. A V2V is expected to use as one of alternative communication methods in a disaster. Because many people escape to the safe area by cars in a disaster, the traffic jam may prevent an 
emergency vehicle from rapid arrival to its destination. In order to pass an emergency vehicle smoothly, some roads are designated in emergency routes. However, emergency routes are not announced for a long time just after a disaster occurrence. For example, it takes about 1 day for designation in emergency route at The Great East Japan Earthquake 2011 [1].

Also, there are some roads impossible to pass due to the collapse of trees, poles and buildings in disasters. Under such circumstances, emergency vehicles such as ambulances and missed cars must arrive at their destinations as soon as possible in order to rescue the injured or sick and to extinguish the building. In many existing studies, delivery plan for the emergency vehicles in the shortest time is proposed in [2] [3] [4] [5]. However, the congestion of the road immediately after the occurrence of the disaster and the existence of the road which became impossible to pass cannot be grasped or predicted before the occurrence of the disaster. The arrival of the emergency vehicle may be delayed. For this reason, it is necessary to quickly acquire the congestion state and information on the road where the emergency vehicle cannot pass without depending on the fixed infrastructure immediately after the occurrence of the disaster. Moreover, a method to dynamically determine the routes of the emergency vehicles is based on this information.

In this paper, in order to design a travel route allowing emergency vehicles to arrive at their destination in as short a time as possible, we propose a method for collecting road conditions in real time using inter-vehicle communication while the emergency vehicle travels toward the destination and dynamically determines the route according to the latest information. A rectangular area including the current location and destination of the emergency vehicle is set on a map as the information collection range. Within this range, we assume that there is enough vehicle capable of wireless communication with map information on the clock and information collection range. The information collected from each vehicle in which the emergency vehicle is in the information collection range is the current location of the vehicle and the location and time of the intersection that passed in the past (we call vehicular information). Each vehicle that has received a query transmitted from the emergency vehicle transmits the latest vehicular information to the emergency vehicle. Emergency vehicles periodically send queries in order to constantly obtain the latest vehicular information. In order to achieve our objective, the following two problems must be solved.

- How to efficiently collect information on emergency vehicles.

- How to construct a travel route of an emergency vehicle by the collected information.

In order to solve these problems, we propose Information gathering algorithm and Route decision algorithm, respectively.

The information collection algorithm is an algorithm for emergency vehicles to efficiently collect vehicle information. This algorithm transmits queries incorporating planned travel route information of emergency vehicles from emer- 
gency vehicles to other vehicles in the target area to as many vehicles as possible with as few hops as possible. The vehicles who received the query refer to the planned travel route of the emergency vehicle and transmit the vehicle information such that the own vehicle information passes on the route.

Route decision algorithm is an algorithm for determining a route arriving at a destination in as short a time as possible. This algorithm predicts traffic congestion status of each road and impassable roads from the vehicular information gathered by Information gathering algorithm.

We describe the related works in Section 2. The problem formulation is described in Section 3. The proposed method is explained in Section 4. The simulation results and considerations are described in Section 5. Finally, we conclude this paper in Section 6.

\section{Related Works}

Toyota Motor Corporation's service called Passable route map [6] during the 2011 Great East Japan Earthquake and the 2016 Kumamoto Earthquake has been put into practical use in the system for presenting traffic conditions at a disaster. However, because it is a service that provides information on the Internet, it cannot be used if the Internet cannot be used. Since the update frequency is one hour and the real time property is low, it is difficult to respond instantly to changes in road conditions. For this reason, we focused on inter-vehicle communication in order to grasp road conditions in real time using only ad-hoc networks.

Inter-vehicle communication is a communication method in which vehicles communicate directly with each other without the infrastructure installed in advance such as a roadside device. Since vehicles may move at high speed, an inter-vehicle communication has a problem in the connectivity of communication. There are many studies for exchanging information via inter-vehicle communication where communication connectivity is not guaranteed. Wischhof et al. proposed a method to predict the congestion of roads by exchanging vehicle position information and speed information when a vehicle enters an oncoming vehicle's communication range [7]. Wischhof et al. proposed UMB that is a method to rapidly deliver information [8]. UMB reduces the number of hops by transmitting information to nodes as far as possible by multi-hop communication. However, since these methods use roadside aircraft installed at each intersection, they may not be usable in environments where infrastructure functions are destroyed by disasters. Zhao et al. proposed a method to dynamically make a path of an emergency vehicle [9]. However, we aim to avoid unpassable roads caused by disasters. A mechanism that finds unpassable roads is needed.

There is a DTN (Delay Tolerant Network) as a technique for transmitting information at a high arrival rate to a specific node. In DTN, information arrival rate can be improved instead of allowing some delay of information transmission. However, DTN is not preferable in this problem where immediacy of in- 
formation transmission is required. On the other hand, Young et al. proposed Geocast as a method of transferring data to a specific geographical area [10]. Geocast has high transmission rate and immediacy. It sets the geographical position at the destination of transmission, and sends packets to the nodes existing in the peripheral area in a multi-hop manner. When this method is used as a method of information gathering of this problem, it is only necessary to regularly set the current location of the moving emergency vehicle as a geographical position. Each vehicle transmits the vehicular information toward the updated point of the emergency vehicle. Therefore, Geocast can be applied to this problem.

In order to dynamically determine the moving route of an emergency vehicle, a new method for quickly collecting information from each vehicle via inter-vehicle communication in the event of a disaster is required. In this paper, we propose the method for efficiently transmitting queries to each vehicle and collecting vehicular information of each vehicle in order to let the emergency vehicle get road status. We also propose the method for making the emergency vehicle rapidly arrive its destination by dynamically determining its moving route based on the latest vehicular information collected while the emergency vehicle traveled.

In this paper, we propose a method to make a path of an emergency vehicle dynamically by collecting travel histories of other cars. Our method can find and avoid unpassable roads by analyzing travel history data.

\section{Problem Formulation}

In this section, we describe the cars, emergency vehicles, assumed environments for roads, definition formulas for vehicles, formulation of the target problem and the approach to solution in this research.

\subsection{Target Environment}

Since there is a possibility that the equipment installed at a fixed position in advance such as mobile phone base station, fixed telephone, road side machine, etc. may have been destroyed, this research targets the environment where data transmission is performed only by inter-vehicle communication. It is assumed that a traffic jam occurs due to the confusion of people who evacuate using a car and roads that cannot be passed exist due to pole and tree collapse. We set a rectangle area $F$ that includes start point of the emergency vehicle and its destination as shown in Figure 1 . There are $N_{\text {all }}$ cars and only 1emergency vehicle in $F$. The emergency vehicle collects vehicular information from $N\left(<N_{\text {all }}\right)$ cars that have radio communication devices and maps. We assume that there are no intersections over five roads on the map. The traveling direction at the intersection of the emergency vehicle and each vehicle is at most three directions of right turn, straight, and left turn, as Figure 2. 
$\mathrm{F}$

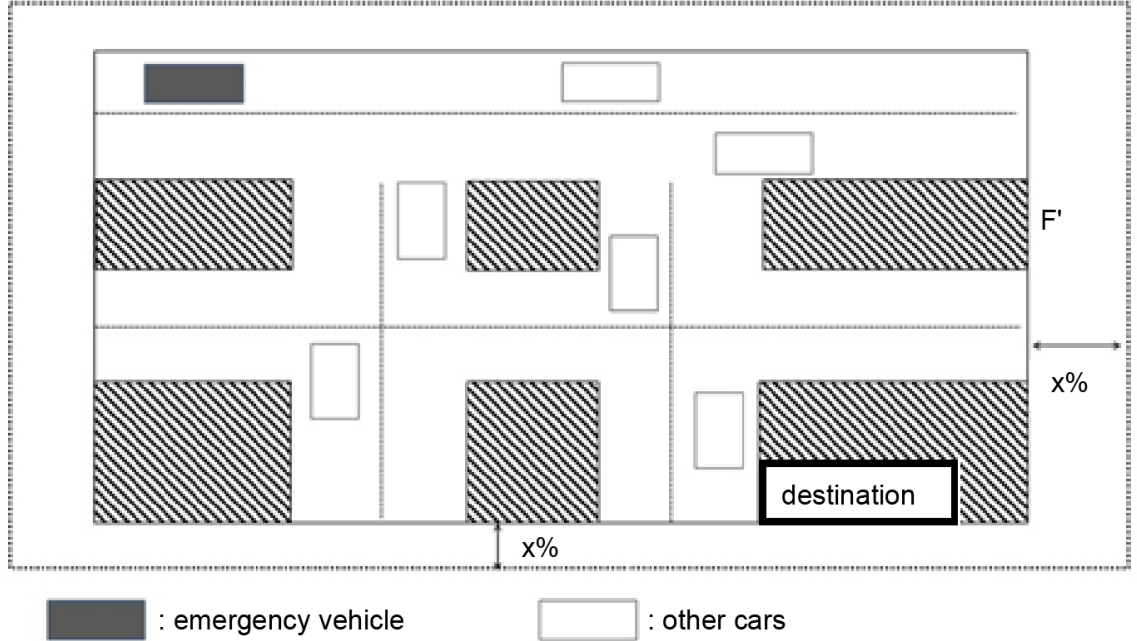

Figure 1. Target field.

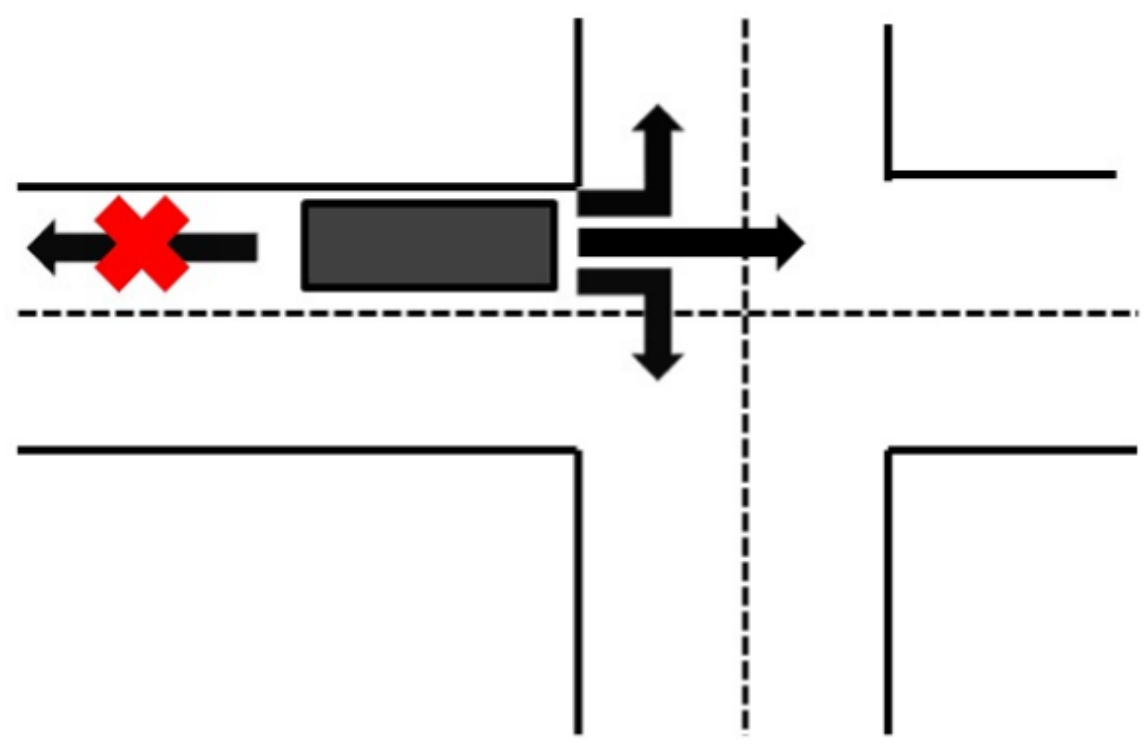

Figure 2. Traveling direction for each vehicle.

Let $r_{E V}$ and $r_{V i}$ denote the communicable range of emergency vehicle and car $V_{i}$, respectively. When the emergency vehicle moves out from the departure point, its route to the destination is set in advance using the existing car navigation system. The initial route determined at this time is the shortest route from the departure point to the destination. Then, the emergency vehicle periodically broadcasts the query to each vehicle in the area $F$ by multi-hop communication. A car that has been received the query from emergency vehicle sends two kinds of information to the emergency vehicle, its current position and passage time of intersections passed in the past. Also, vehicles outside $F$ do not transmit vehicle information even if they receive information from emergency vehicles. The emergency vehicle judges that the road which no vehicles have passed through is not passable, and constructs a traveling route not using that road. The query 
transmitted by the emergency vehicle and the vehicular information transmitted by cars that received the query to the emergency vehicle are as follows. Information included in the query transmitted by the emergency vehicle is as follows.

- Scheduled traveling route of emergency vehicle

- Target area $F$

- ID of the query

Vehicular information each car has includes as following data.

- Current sender's position

- Passage time of intersections passed in the past

\subsection{Definitions}

Let $\left\{C_{1}, C_{2}, \cdots, C_{n}\right\}$ denote the set of all intersections in $F$. Here, the departure point is denoted by $C_{0}$. Let $\left\{C_{i 1}, C_{i 2}, \cdots, C_{i k}\right\}(0 \leq k \leq n)$ be the set of intersections where the car $V_{i}$ passed. This is called the travel history of the vehicle $V_{i}$ Let $T_{C_{i x}}$ be the time when the vehicle $V_{i}$ passed through the intersection $C_{i x}$. The vehicular information of the car $V_{i}$ that has travel history $\left\{C_{i 1}, C_{i 2}, \cdots, C_{i k}\right\}$ is represented by $\left\{T_{C_{i 1}}, T_{C_{i 2}}, \cdots, T_{C_{i k}}\right\}$.

The time $T_{\left(C_{i k}, C_{i l}\right)}$ required for the car $V_{i}$ to pass between the adjacent intersections $C_{i k}$ and $C_{i l}(k<I)$ can be expressed by the following equation.

$$
T_{\left(C_{i k}, C_{i l}\right)}=T_{C_{i l}}-T_{C_{i k}}
$$

Assuming that the number of cars to which the vehicle information has been delivered to the emergency vehicle at time $t$ is $N^{\prime}\left(N^{\prime} \leq N\right)$, the vehicular information $E V_{\text {info }}(t)$ gathered in the emergency vehicle by the time $t$ can be expressed as follows.

$$
E V_{\text {info }}(t)=\bigcup_{i=1}^{N^{\prime}} \bigcup_{u=1}^{j}\left\{T_{C_{i 1}}, T_{C_{i 2}}, \cdots, T_{C_{i u}}\right\}
$$

However, $j$ is the number of intersections present in the travel history of each car. When collecting vehicular information from multiple vehicles, it is possible to calculate the average passing time between each intersection. Assuming that the number of vehicles that passed the intersection $\left(C_{k}, C_{l}\right)$ by the time $t$ is $N_{t}^{\prime}$, the average passing time between the intersections can be represented by the following formula

$$
T_{\text {ave }}\left(C_{k}, C_{l}\right)=\frac{1}{N^{\prime}} \sum_{i=1}^{N^{\prime}} T_{\left(C_{i k}, C_{i l}\right)}
$$

Here, if $N^{\prime}=0, T_{\text {ave }}\left(C_{k}, C_{l}\right)=\infty$.

\subsection{Objective Function}

In a disaster, the emergency vehicle must arrive at the destination as soon as possible, and the objective function of the target problem is expressed by the following equation.

$$
\operatorname{Minimize}\left(\operatorname{cost}\left(E V_{\text {info }}(t), E V \cdot p o s(t), \text { Dest.pos }\right)\right)
$$


Here, EV.pos $(t)$ and Dest.pos represents the position of the emergency vehicle at time $t$ and the position of the destination, respectively. Also, the function $\cos t()$ represents the time taken for the emergency vehicle to travel through a route calculated from the collected vehicle information and the current position of the emergency vehicle and the position of the destination.

In order to make this time as short as possible, it is necessary to determine the route that arrives earliest from the dynamically changing road conditions at that time. In order to know the latest road condition, it is necessary for the emergency vehicle to gather the latest vehicular information from each vehicle at regular intervals, and to construct a route to arrive at the destination at the earliest time based on the vehicular information.

The inputs of our target problem are the target area $F$, the set of intersections $\left\{C_{1}, C_{2}, \ldots, C_{n}\right\}$, the emergency vehicle, and cars $V_{1}, V_{2}, \ldots, V_{N}$ that have travel history. The proposed method makes the emergency vehicle efficiently collect the vehicular information and estimates the time taken for passing through each intersection. Finally, the proposed method outputs a route that can arrive from the current location of the emergency vehicle to the destination in as short a time as possible.

\section{Proposed Method}

In this section, we describe a proposed method for collecting more vehicular information on emergency vehicles in order to create a route for emergency vehicles to arrive at the destination as soon as possible. The proposed method consists of two algorithms, Information gathering algorithm (IGA) and Route construction algorithm (RCA), because the emergency vehicle periodically collects vehicle information and dynamically changes the travel route. IGA is an algorithm for efficiently gathering vehicle information in order to grasp the latest road condition from the emergency vehicle to the destination, and it is applied at constant intervals. RCA is an algorithm for constructing a route for the emergency vehicle to arrive at the destination as soon as possible on the basis of the information gathered by the IGA, and this is also applied at regular intervals.

\subsection{Information Gathering Algorithm (IGA)}

\subsubsection{Overview}

IGA makes the emergency vehicle efficiently transmit queries to each car. IGA also makes a car that received the query send its vehicular information to the emergency vehicle.

At first, the emergency vehicle send the query to the cars in the communicable range of the emergency vehicle $r_{E V}$. The query consists several kind of information such as $F$, the query ID, and the initial route of the emergency vehicle determined by car navigation system.

Each car that newly receives this query sends its current location and travel history to the emergency vehicle. Among the secars, the car most distant from 
the emergency vehicle in each direction (left, front, back, and right direction toward the traveling direction of the emergency vehicle) also relays the query to the cars in the communicable range of it. Each car that receives the relayed query also sends its vehicular information. By continuing these processes, the emergency vehicle broadcasts the query.

The cars that relay the query transmitted by the car $V_{i}$ are denoted by $V_{\left(V_{i}, L\right)}$, $V_{\left(V_{i}, C\right)}, V_{\left(V_{i}, B\right)}, V_{\left(V_{i}, R\right)}$ as Figure 3. Here, $V_{\left(V_{i}, L\right)}, V_{\left(V_{i}, C\right)}, V_{\left(V_{i}, B\right)}$, and $V_{\left(V_{i}, R\right)}$ are the cars most distant from $V_{i}$ in left, front, back, and right direction.

All of cars that receive the query send their vehicular information to the emergency vehicle via multi-hop communication. The car can know the expected traveling route of the emergency vehicle by receiving the query. The car finds the point on the expected traveling route of the emergency vehicle and closest to its current position. The car also sends its vehicular information by Geocast manner to the point. Then, the vehicular information is delivered along with the expected traveling route of the emergency vehicle to the emergency vehicle.

The emergency vehicle collects vehicular information for time $T_{\text {route }}$ and calculate a new traveling route by RCA (described later). When the calculation terminates, the emergency vehicle broadcasts a new query with the updated traveling route.

We describe three kinds of algorithms for the emergency vehicle, the cars that relay the query, and the other cars.

\subsubsection{Algorithm for the Emergency Vehicle}

Detail of algorithm for the emergency vehicle is as follows. Figure 4 shows the flowchart.

1) An emergency vehicle constructs a traveling route by RCA from gathered vehicular information. If there is no vehicular information, the shortest route from current position to the destination is output using the car navigation system of the emergency vehicle.

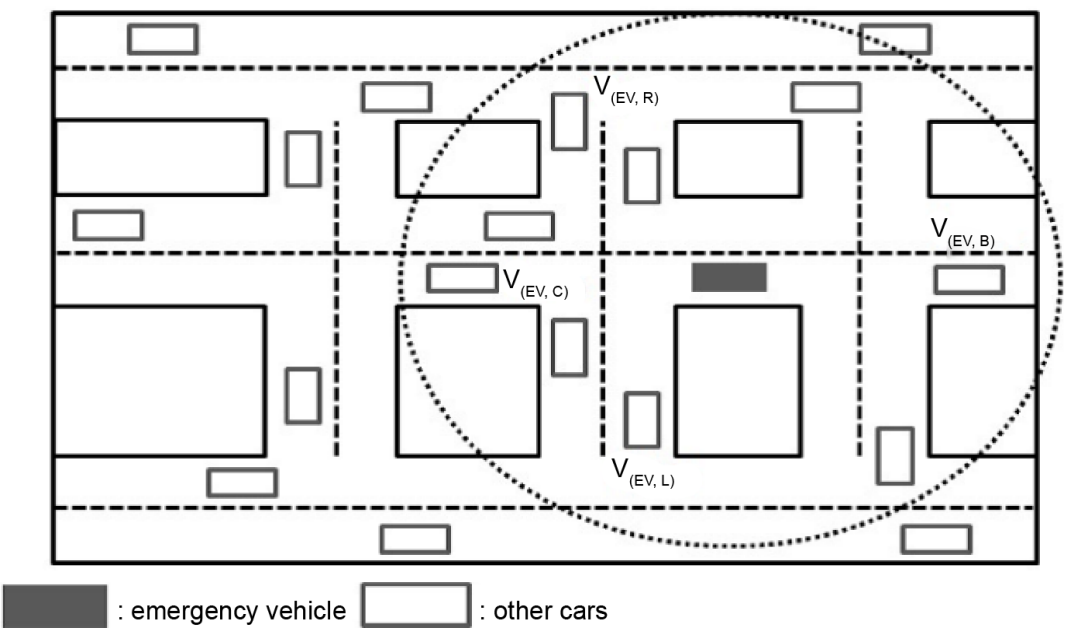

Figure 3. Example of $V_{(E V, L)}, V_{(E V, C)}, V_{(E V, B)}, V_{(E V, R)}$. 


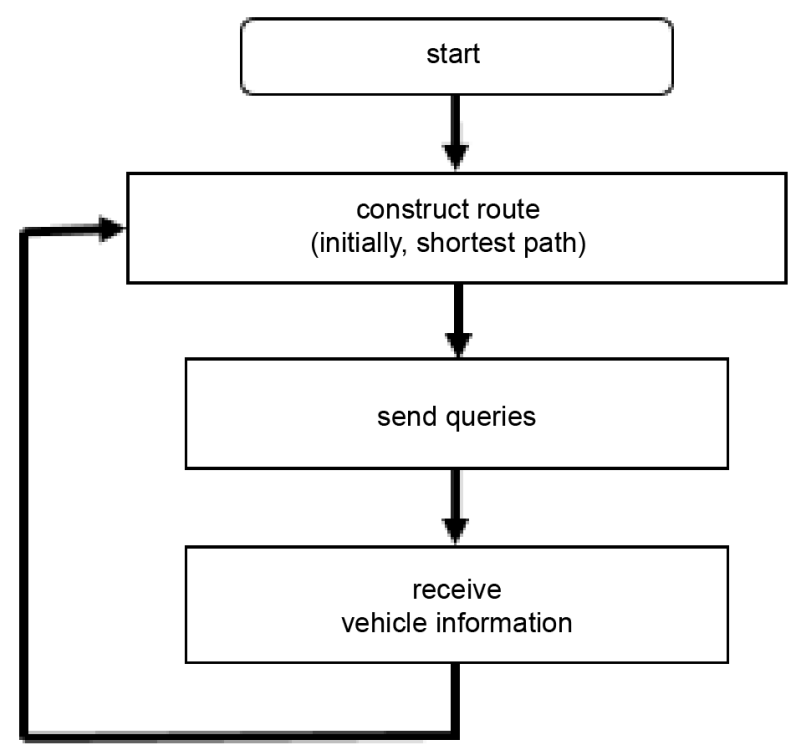

Figure 4. Algorithm flowchart for emergency vehicle.

2) The emergency vehicle broadcasts the query.

3) When the emergency vehicle receives a reply from the vehicle in $r_{E V}$, the emergency vehicle sends acknowledgement. The emergency vehicle decides $V_{(E V, L)}, V_{(E V, \mathcal{O})}, V_{(E V, B)}$, and $V_{(E V, R)}$ and inform them to relay the query.

4) Wait for $T_{\text {route }}$ and go to Step (1).

\subsubsection{Algorithm for the Relaying Cars}

Let $S$ be the set of relaying cars that propagate the query to other cars. The initial $S=\left\{V_{(E V, L)}, V_{(E V, C)}, V_{(E V, B)}, V_{(E V, R)}\right\}$ is determined when the emergency vehicle is initially made the above step (3). Each $s \in S$ performs this algorithm.

1) If the car $s$ is selected to a relaying car of propagating query, $s$ find $\left\{V_{(s, L)}\right.$, $\left.V_{(s, C)}, V_{(s, B)}, V_{(s, R)}\right\}$.

2) The car $s$ sends its vehicular information to the emergency vehicle by both of Geocast and Unicast (the detail is described in the next subsection).

\subsubsection{Algorithm for Sending Vehicular Information}

In this subsection, an algorithm for sending vehicular information is via Geocast and Unicast. If vehicular information is sent by only Geocast, the destination vehicle may move during the relay of the vehicular information. In order to solve this problem, our algorithm let the vehicular information firstly be sent on the traveling route of the emergency vehicle and be delivered along the route.

In this subsection, $v$ represents a car that wants to send its vehicular information to the emergency vehicle. The expected traveling route of the emergency vehicle is also represented by $R$. List $L_{V}$ represents the set of query IDs that the car $v$ has been received.

1) If $v$ 's received query ID $z$ is in $L_{v}$ algorithm terminates.

2) If $v$ is on $R, u=v$ and go to Step (3).

3) $v$ send its vehicular information by Geocast to the destination area where 
the point on $R$ closest to $v$.

4) The car $u$ on $R$, that has the vehicular information, transmits it to the emergency vehicle by Unicast targeting only the car on $R$

5) Add $z$ into $L v$.

\subsection{Route Construction Algorithm (RCA)}

\subsubsection{Overview}

In this section, we describe about RCA. Emergency vehicles estimate road conditions from the vehicular information gathered by IGA and determine the traveling route to arrive at the destination in as short a time as possible by RCA. RCA solve the problem with the Dijkstra method [11] as the shortest path problem in directed graphs where an intersection is a vertex, a road between intersections is an edge, a traveling direction of a road is a direction, an average transit time of each intersection is a weight of edge, and a map is a directed graph, as shown in Figure 5.

In the vehicular information gathered to the emergency vehicle, let $C_{\text {info }}$ be the set of intersections connected from the next intersection where the emergency vehicle passes, and $C_{\text {noinfo }}$ be the set of intersections not connected.

RCA firstly finds the average time taken for passing between each intersection included in $C_{\text {infor }}$. The average time taken for passing between intersections $C_{k}$ and $C_{l}$ is represented by $T_{a v e}\left(C_{k}, C_{l}\right)$. RCA estimates the time taken for arriving to each intersection in $C_{\text {info }}$. However, for intersections where vehicle information is not gathered, we cannot define the weight of edges as in (1) because we do not know the average time to pass. Therefore, road information prior to the disaster occurring in the emergency vehicle is defined as the weight of the edge as the average transit time between each intersection. For each intersection $C_{\text {end }}{ }^{2} C_{\text {info }}$ and having edges connecting $C_{\text {info }}$ and $C_{\text {noinfo }}$ without a point close to the destination among the adjacent intersections, RCA calculates the fastest route to the destination using the Dijkstra method starting from $C_{\text {end }}$ and make those times be the weights of vertices of each $C_{\text {end }}$. Figure 6 shows an example of $C_{\text {info }}$ and $C_{\text {noinfo }}$ areas.

RCA combines these $C_{\text {info }}$ and $C_{\text {noinfo }}$ paths, and finds the combination that minimizes the travel time. If the current traveling route takes more time than the

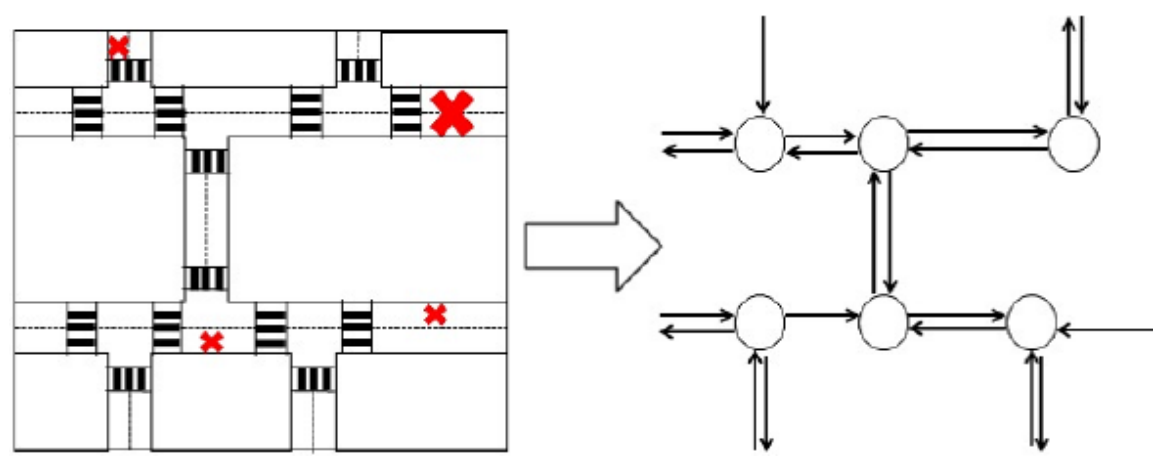

Figure 5. Directed graph. 
new route, the emergency vehicle selects the new route. A flowchart of RCA is shown in Figure 7.

\subsubsection{Detail of RCA}

1) Route from the departure point of the emergency vehicle to the intersection belonging to $C_{\text {info }}$

We assume that intersections in $C_{\text {info }}$ are vertices, roads between adjacent intersections are edges, $T_{a v e}\left(C_{k}, C_{l}\right)$ that represents average travel time between adjacent intersections $C_{k}$ and $C_{l}$ is a weight of edge, and the time for traveling from the current position of the emergency vehicle to the intersection $C_{k}$ is the evaluation value of $C_{k}$

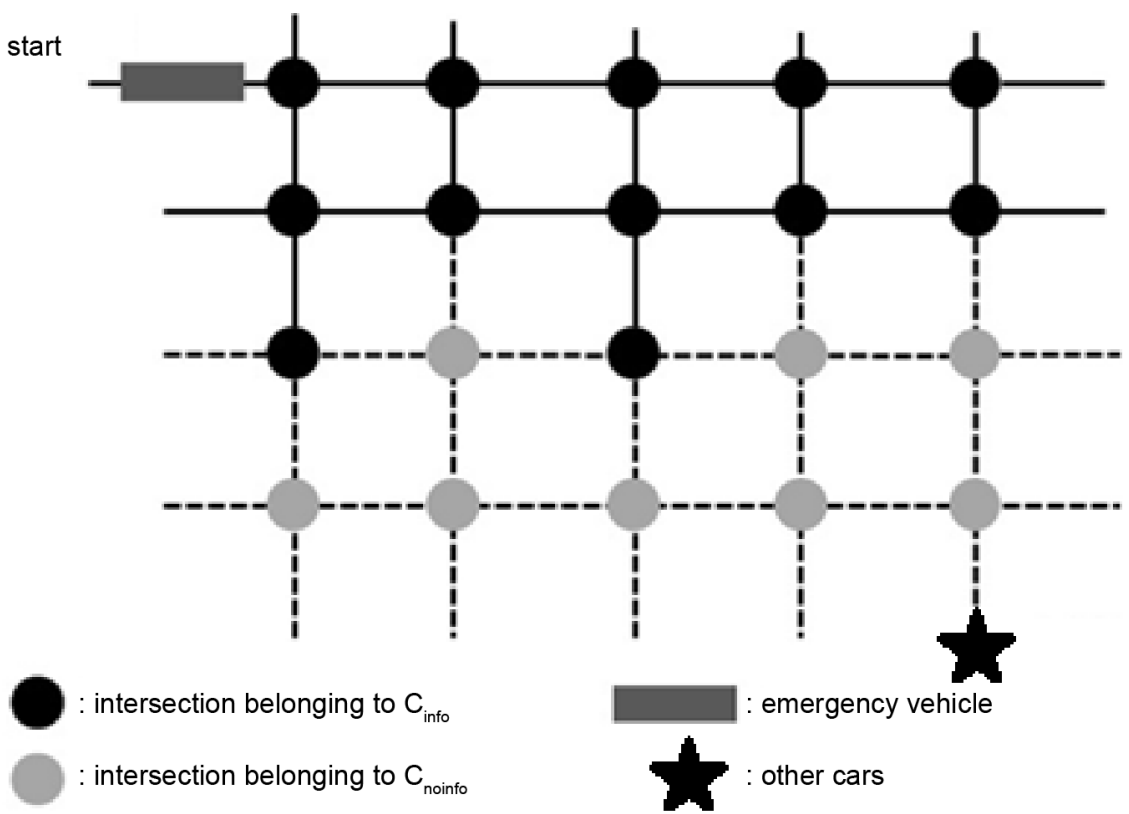

Figure 6. $C_{\text {info }}$ and $C_{\text {noinfo }}$ areas.

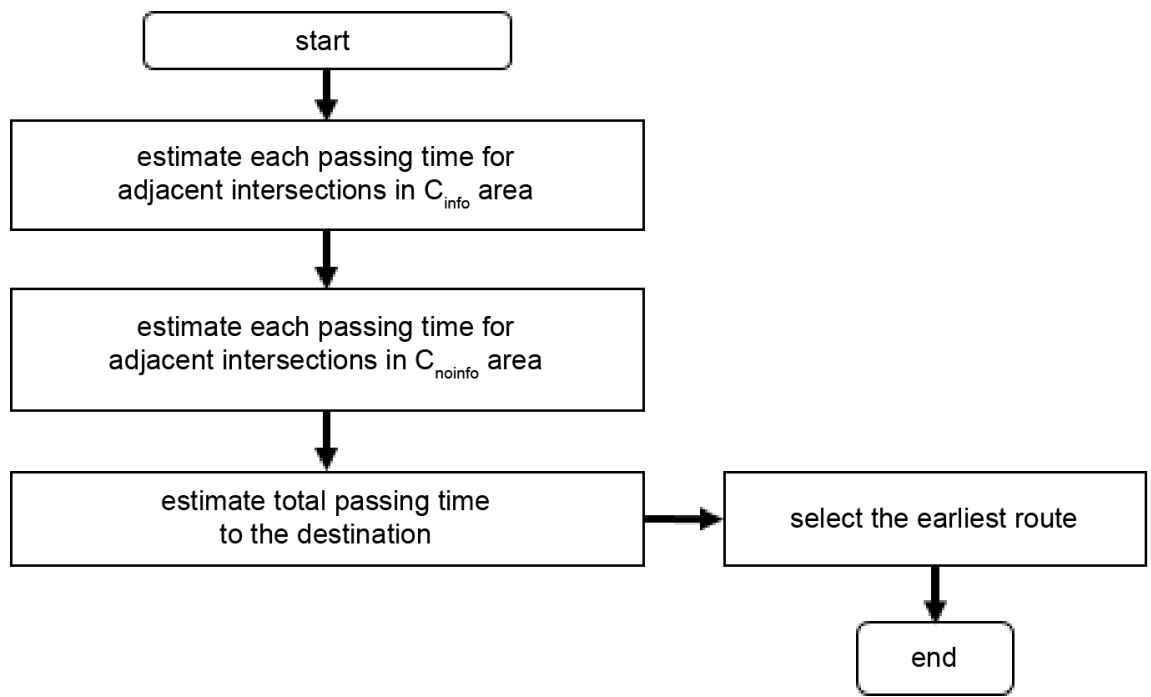

Figure 7. RCA flowchart. 
a) Let point $p$ be the departure point of the emergency vehicle and the weight of each point is set to 1 . At this time, the weight of the point $p$ is 0 . The weights of points other than the start point are assumed to be uncertain.

b) If the weight of the point $q$ adjacent to $p$ is 1 , update the weight of $q$ to the weight of the edge between $p$ and $q$. Otherwise, update the weight of $q$ when the sum of the weight of the edge and the weight of $p$ is smaller than the existing weight of $q$. Set $p$ to the newly updated point, and repeat from step (2). If there is no point where the weight is newly updated, this algorithm terminates.

2) Route from the departure point of the emergency vehicle to the intersection belonging to $C_{\text {info }}$

For the $C_{\text {noinfo }}$ area, RCA calculates the average transit time from the existing road information that the emergency vehicle has before the occurrence of the disaster and set that time as the weight of the edge.

Let $C_{\text {end }}$ be the set of vertices where the intersection belonging to $C_{\text {noinfo }}$ is adjacent to the intersection included in $C_{\text {info }}$ and the vertex of $C_{\text {info }}$ nearer to the destination does not exist than itself (Figure 8). For all $p 2 C_{\text {end }}$ the algorithm explained above runs. Thus, we can get the weight of each vertex. For all of $p 2$ $C_{\text {end }}$, RCA investigates the shortest arrival time to the destination via $p$ by Dijkstra algorithm. Finally, RCA selects the shortest route as Figure 9 shows.

\section{Simulation}

In this simulation, the emergency vehicle moves to the destination while dynamically changing the route from the collected vehicular information. We compared the proposed method with Geocast [10] on the number of vehicular information and intersection information collected until the emergency vehicle arrives at the destination and the time it takes to arrive at the destination. The

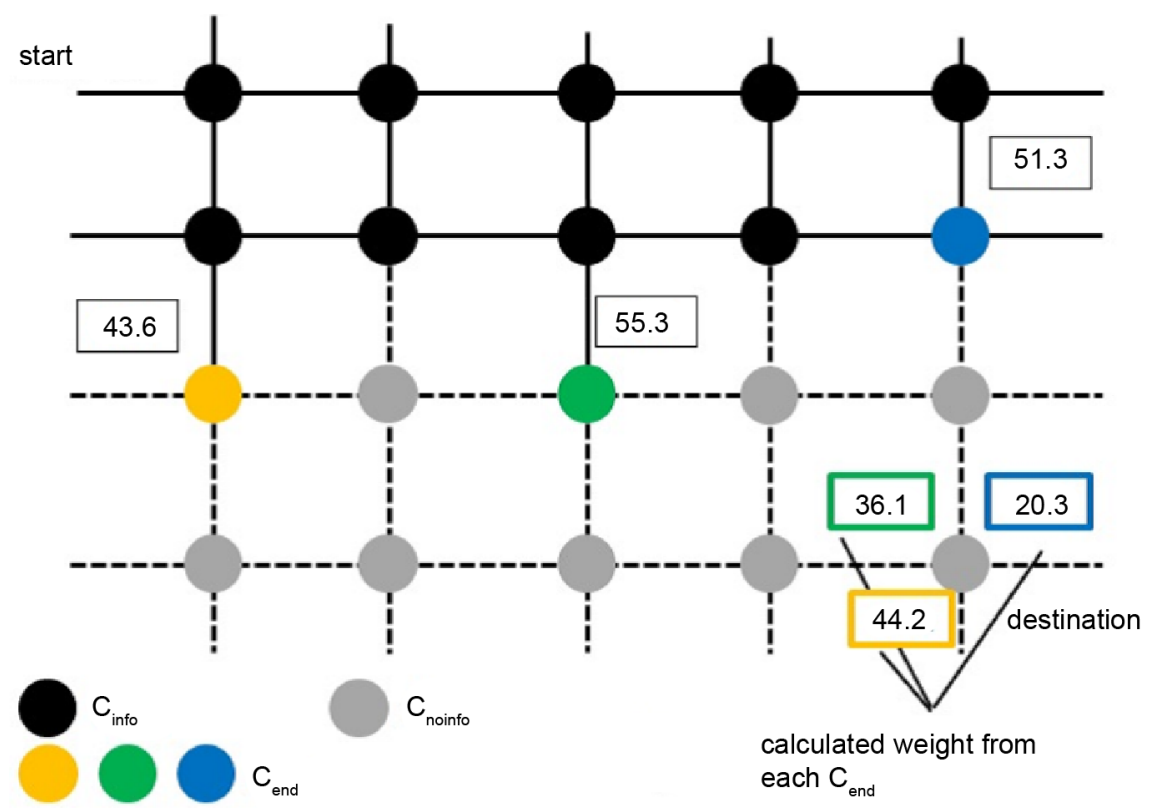

Figure 8. Example of $C_{\text {end }}$ 


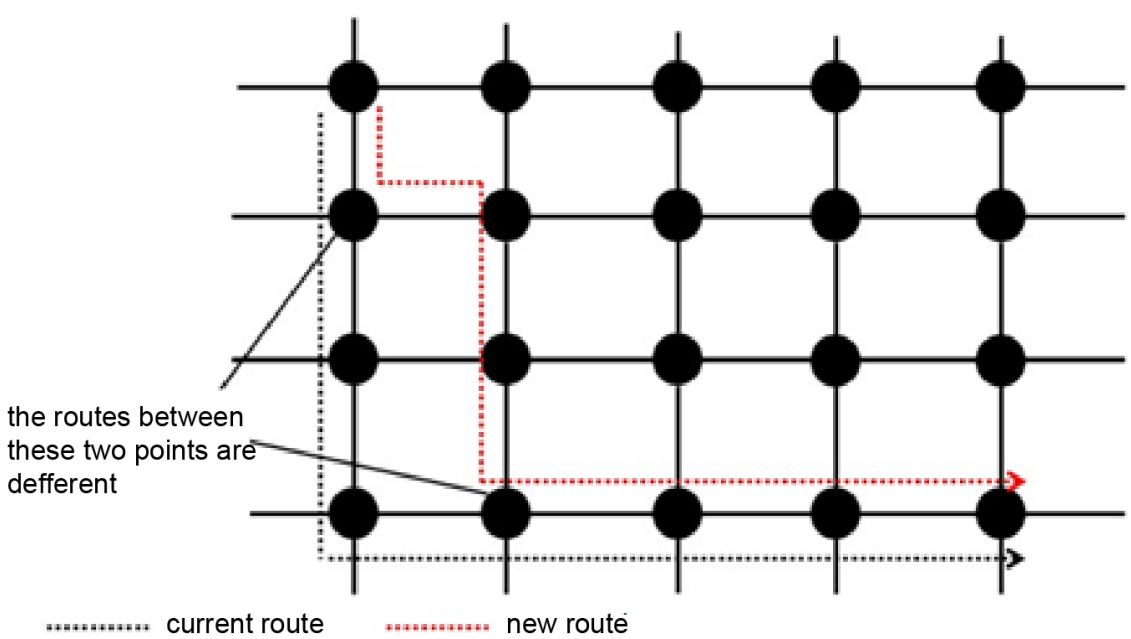

Figure 9. Modified route.

intersection information is the passing time of each intersection in the travel history of the car. For example, suppose that vehicular information is collected from two cars in an emergency vehicle. When the vehicle $v_{1}$ has a travel history at three intersections and the vehicle $v_{2}$ has a travel history at two intersections, the number of vehicular information is 2 and the number of intersection information is 5 . As the number of vehicular and intersection information increases, the reliability of road passage time prediction also increases, so the larger one is a better result.

\subsection{Settings}

In this simulation experiment, a rectangular area of $720[\mathrm{~m}]$ north, $700[\mathrm{~m}]$ east, 130 [m] south from the Nakagyo fire department in Kyoto is targeted (Figure 10). Assuming that there are buildings in all areas excluding roads in the target area, each vehicle can transmit and receive information only to the vehicle on the extension line of the road where the vehicle is located. Also, for all vehicles in the target area excluding the emergency vehicle, the departure point and the destination are randomly determined, and the departure time is randomly set. At the start of the simulation, each vehicle has its travel history from the departure point to the current position, and travels toward the destination simultaneously with the start of the simulation. Three random places on the road were preliminarily set as impossible to pass. There is no upper limit on the number of vehicle information that each vehicle can record. If each vehicle cannot communicate for $20 \mathrm{sec}$, it discards vehicular information relayed by itself.

The parameters are shown as follows.

- Target field: $860 \_700[\mathrm{~m}]$

- The number of cars: 600

- Length of each car: $4[\mathrm{~m}]$

- Radius of communicable range: 40 [m]

- Time for communication completion: 0.2 [s] 


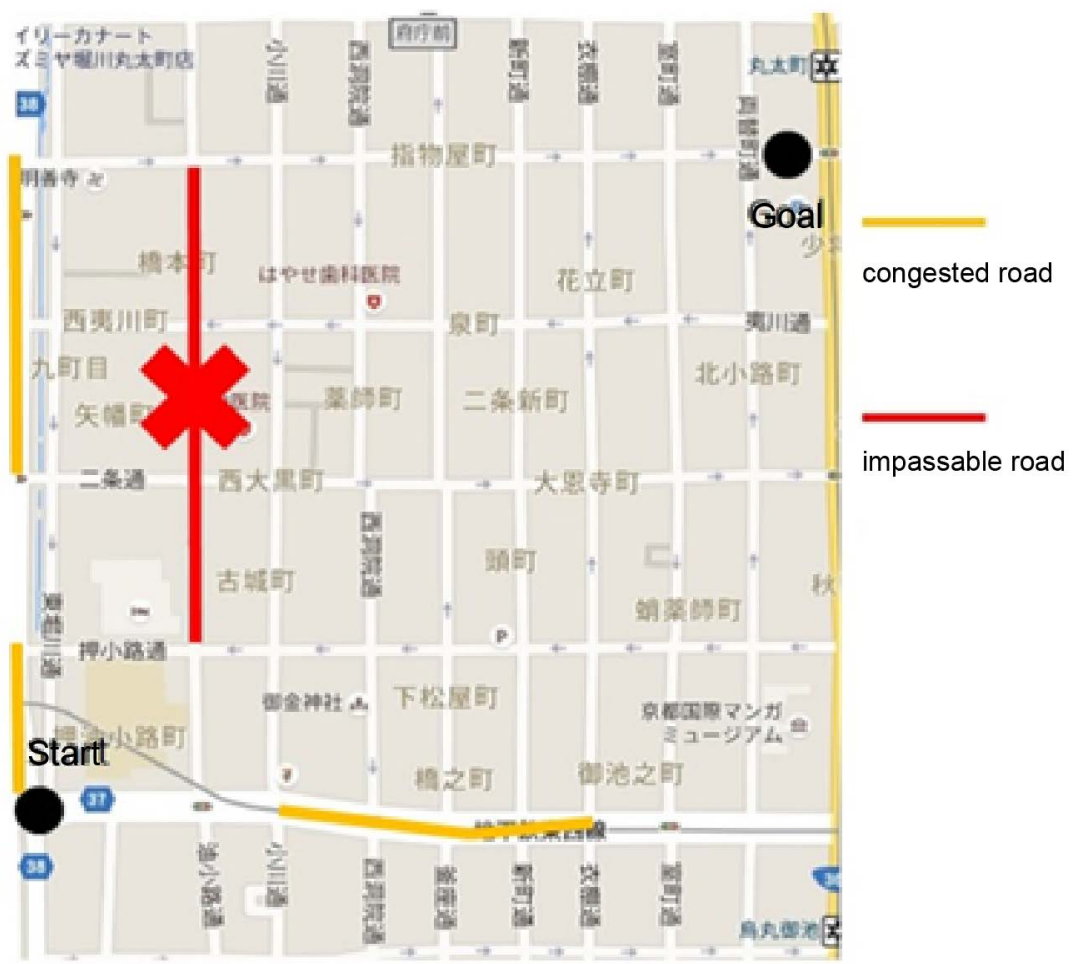

Figure 10. Target map.

\section{Comparison Method: Geocast}

The method of sending the query is the same as the proposed method, and Geocast is used as the method for sending vehicular information to the emergency vehicle. Geocast is a method that sets a transmission target area for delivering information to that area. In this experiment, a range of $300[\mathrm{~m}]$ forward from the traveling direction of the emergency vehicle is set as the transmission target area. The transmission target area at the time of departure of the emergency vehicle is as shown in Figure 11. It is updated based on the current location when the emergency vehicle transmits the next query. The determination of the movement route of the emergency vehicle is the same as the proposed method.

\subsection{Simulation Result}

Figure 12 and Figure 13 show the number of vehicular information and the number of intersection information collected in the emergency vehicle at each time (second). These are the average values of 20 trials.

We confirmed that both information is gathered more in the proposed method. At each time, the proposed method was able to collect about $12.5 \%$ more information than Geocast. The arrival time to the destination of the emergency vehicle was 224 seconds for the proposed method and 253 seconds for Geocast. There were no cases where it was impossible to judge where traffic could not pass and the destination could not be reached. Also, when the emergency vehicle changes its route more than once, the average time it takes to change the route for the first time is 12 seconds for the proposed method and 25 seconds for Geocast. 


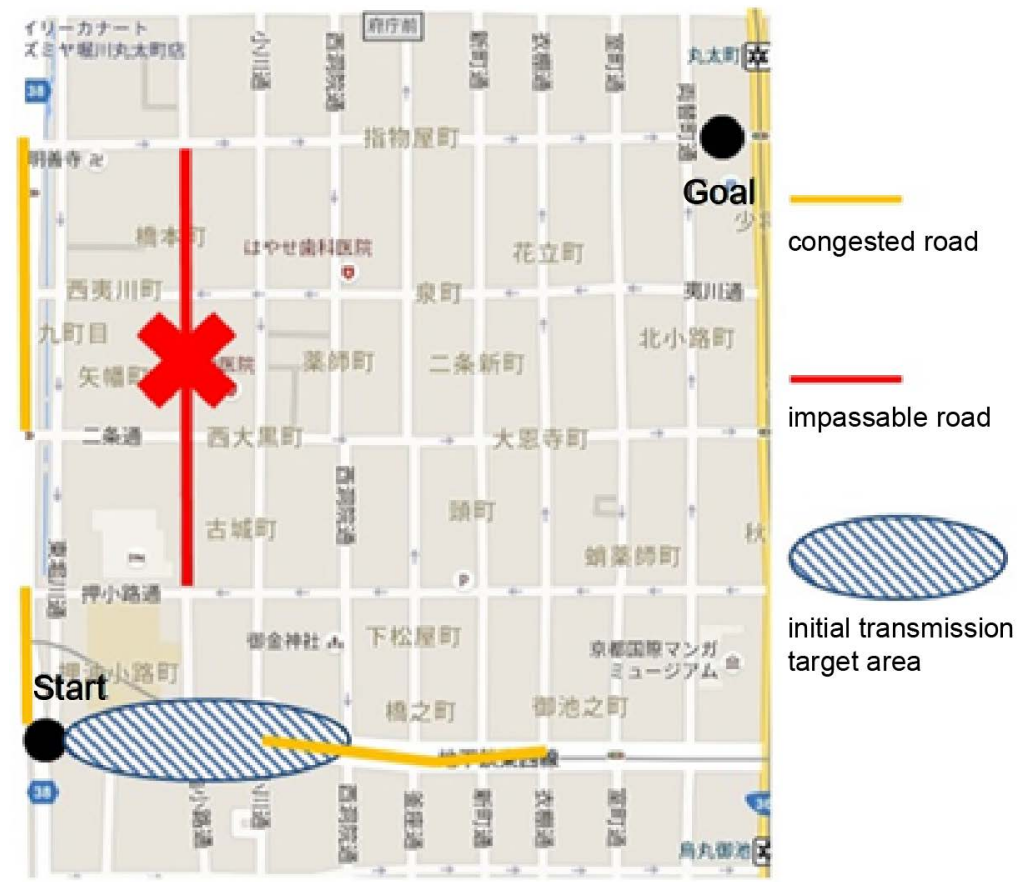

Figure 11. Transmission target area of Geocast.

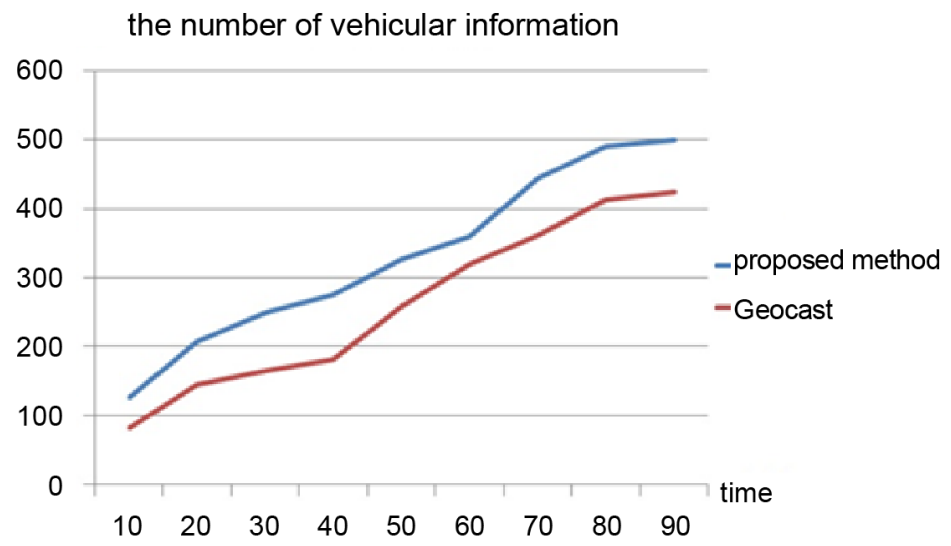

Figure 12. The number of vehicular information the emergency vehicle obtained.

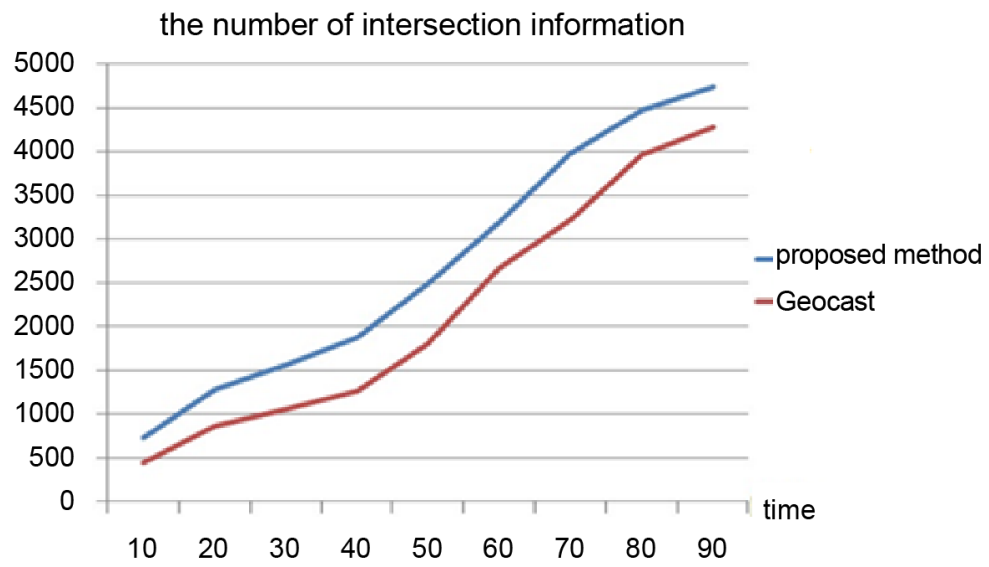

Figure 13. The number of intersection information the emergency vehicle obtained. 
An example of simulation results is shown in Figure 14. The arrival time of the initial route of the emergency vehicle was 181 seconds, however, this does not take into consideration roads that are impossible to pass or congested roads. We confirmed that the route constructed by the proposed method and Geocast avoids roads that cannot pass and congested roads and arrives at the destination as quickly as possible. From Figure 12, we also confirmed that the increase rate of Geocast's information collection is low at time $30-40$ when the emergency vehicle changes the route and passes the road different from the existing route.

According to Figure 15, for example, at the time of 40, the proposed method can collect much intersection information. The reason for this is that in the proposed method, the vehicle that receives the emergency vehicle's query can refer to the planned travel route of the emergency vehicle, so the possibility of transmitting the information to the emergency vehicle becomes high. On the other hand, if the changed route is distant from the target transmission area, Geocast may not transmit information to the emergency vehicle. There is a difference in the number of information collected in the emergency vehicle immediately after the route change. Therefore, it can be said that the proposed method is effective in environments where road conditions frequently change and the route of emergency vehicles becomes complicated.

\section{Conclusions}

In this paper, we proposed an algorithm that dynamically constructs a route for emergency vehicles to arrive at the destination as soon as possible while grasping

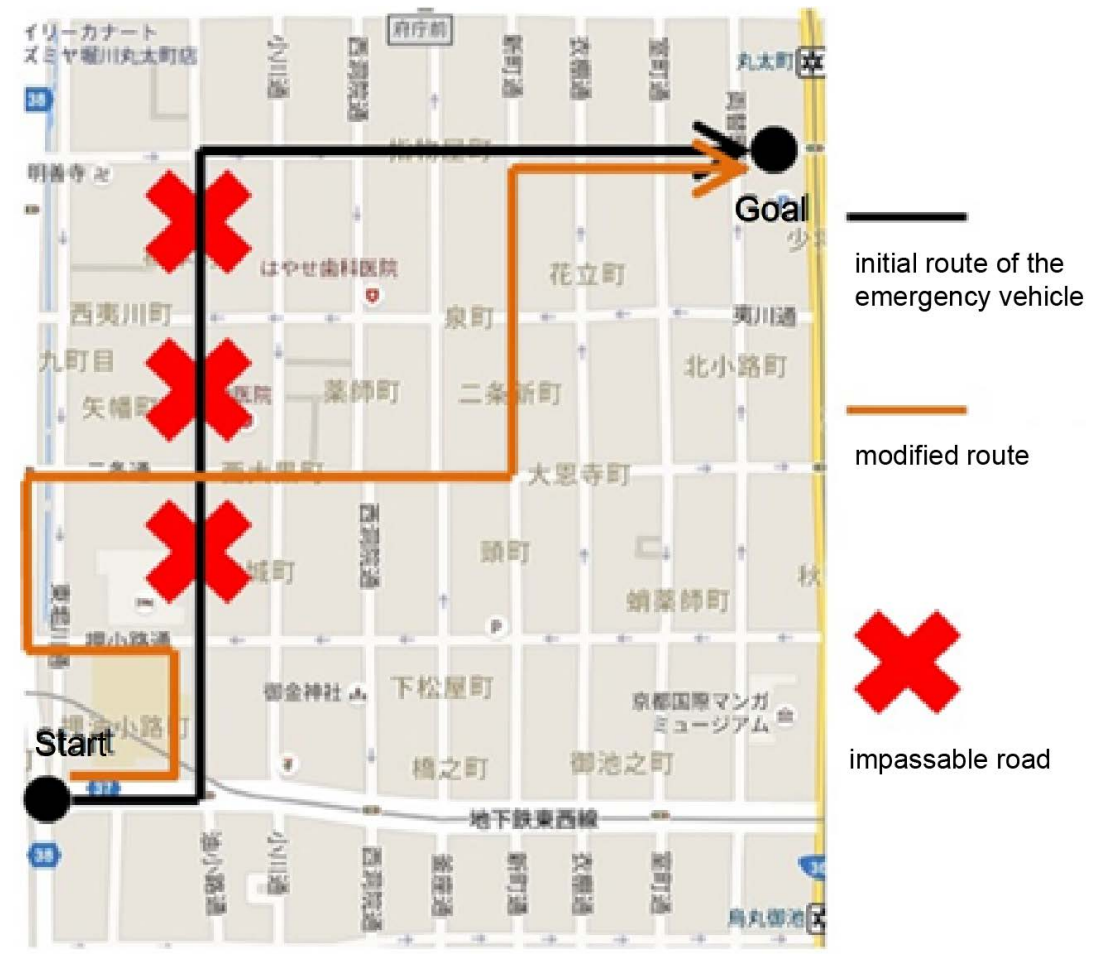

Figure 14. One of simulation results. 


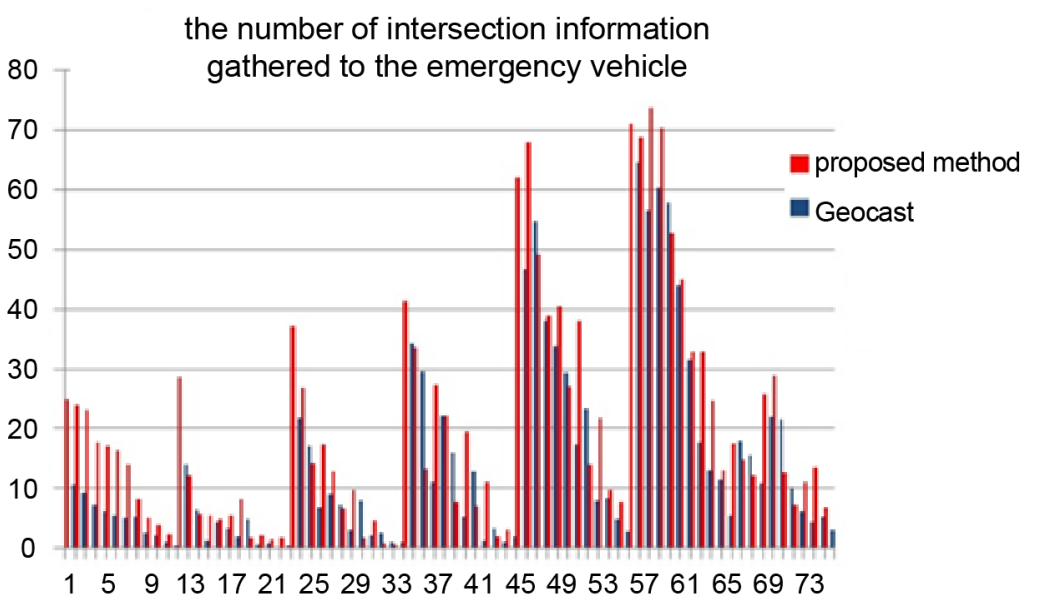

Figure 15. The number of intersection information gathered to the emergency vehicle at time 40 .

the road conditions in real time after a disaster occurrence. The proposed method is constructed by IGA and RCA.

IGA transmits queries from emergency vehicles to vehicles in the target area with as few hops as possible and conveys vehicle information so that each vehicle that received the query passes on the planned travel route of the emergency vehicle.

RCA calculates the average speed of passing through the intersections from the departure point of the emergency vehicle to the destination from the vehicular information of each car received, determines the intersection as a vertex and the road connecting between the two vertices, and constructs a route so that the travel time is minimized by using the Dijkstra method as the shortest path problem in directed graphs. As a result of comparative simulation of the proposed method and existing method Geocast, we confirmed that the proposed method collects vehicular and intersection information about $12.5 \%$ and $11 \%$ more than Geocast, respectively. The proposed method shortens the average arrival time to the destination by about $10 \%$ from Geocast.

In future works, to find impassable roads and to send information packets efficiently, we consider that estimation of vehicle density for each area will be needed. So, firstly, we should appropriately define a vehicle density to improve the proposed method.

\section{References}

[1] Japan National Police Agency. (2011) Traffic Regulation Accompanying the Great East Japan Earthquake.

https://www.npa.go.jp/archive/keibi/biki/traffic/koutu_kisei/koutsukisei.pdf

[2] Talarico, L., Meisel, F. and Sorensen, K. (2015) Ambulance Routing for Disaster Response with Patient Groups. Computers and Operations Research, 56, 120-133. https://doi.org/10.1016/j.cor.2014.11.006

[3] Oran, A., Tan, K.C., Ooi, B.H., Sim, M. and Jaillet, P. (2012) Location and Routing Models for Emergency Response Plans with Priorities. Communications in Com- 
puter and Information Science, 318, 129-140.

https://doi.org/10.1007/978-3-642-33161-9_20

[4] Nordin, N.A.M., Zaharudin, Z.A., Maasar, M.A. and Nordin, N.A. (2012) Finding Shortest Path of the Ambulance Routing: Interface of $A^{*}$ Algorithm Using C\# Programming. Proceedings of IEEE Symposium on Humanities, Science and Engineering Research, Kuala Lumpur, 24-27 June 2012, 1569-1573.

https://doi.org/10.1109/SHUSER.2012.6268841

[5] Smitha, S.B., Narendra, K.G., Usha, R.H.V., Divyashree, C.K., Gayatri, G. and Aparajitha, M. (2012) GPS Based Shortest Path for Ambulances Using VANETs. Proceedings of International Conference on Wireless Networks (ICWN2012), 49, 190-196.

[6] Toyota Motor Corporation. (2016) Passable Route Map. https://www.toyota.co.jp/jpn/auto/passable route/

[7] Wischhof, L., Ebner, A. and Rohling, H. (2005) Information Dissemination in Self-Organizing Inter-vehicle Networks. IEEE Transaction on Intelligent Transportation Systems, 6, 90-101. https://doi.org/10.1109/TITS.2004.842407

[8] Korkmaz, G., Ekici, E., Ozguner, F. and Ozguner, U. (2004) Urban Multi-Hop Broadcast Protocol for Inter-Vehicle Communication Systems. Proceedings of the 1st ACM International Workshop on Vehicular Ad Hoc Networks (VANET04), Philadelphia, PA, 1 October 2004, 76-85. https://doi.org/10.1145/1023875.1023887

[9] Zhao, J., Guo, Y. and Duan, X. (2017) Dynamic Path Planning of Emergency Vehicles Based on Travel Time prediction. Journal of Advanced Transportation, 2017, Article ID: 9184891. https://doi.org/10.1155/2017/9184891

[10] Young, B.K. and Nitin, H.V. (1999) Geocasting in Mobile Ad Hoc Networks: Location-Based Multicast Algorithms. Proceedings of IEEE Workshop on Mobile Computing Systems and Applications (WMACSA'99), Washington DC, 25-26 February 1999, 101-110.

[11] Dijkstra, E.W. (1959) A Note on Two Problems in Connexion with Graphs. Numerische Mathematik, 1, 269-271. https://doi.org/10.1007/BF01386390 